\title{
Effects of the putative phospholipid precursors, inositol, choline, serine and ethanolamine, on formation and expansion of rabbit blastocysts in vitro
}

\author{
M. T. Kane \\ Department of Physiology, University College, Galway, Ireland
}

\begin{abstract}
Summary. Rabbit morulae were cultured to blastocysts in various concentrations of the potential phospholipid precursors, myo-inositol, choline, serine and ethanolamine. Serine $(20-2500 \mu \mathrm{M})$ had a significant stimulatory effect on blastocyst formation and blastocyst expansion and inositol (3-375 $\mu \mathrm{M})$ had a significant stimulatory effect on blastocyst expansion. There was no significant stimulatory effect of choline or ethanolamine.
\end{abstract}

Keywords: blastocyst expansion; rabbit; phospholipid precursors

\section{Introduction}

The nutrient requirements for culture of rabbit embryos through the preimplantation period are much more complex than for the mouse (see Kane, 1987, for a review). Amino acids are essential for blastocyst formation and water-soluble vitamins have been shown to be essential for blastocyst expansion (Daniel, 1967; Kane \& Foote, 1970). Kane (1988) investigated which of the 11 vitamins from F10 medium were essential for development of morulae to expanding blastocysts in vitro by omitting vitamins individually from the culture medium; the most drastic reduction in blastocyst expansion was caused by the omission of inositol. Since inositol is a precursor for phospholipid synthesis, it seemed logical to investigate the importance of a group of putative phospholipid precursors (inositol, choline, serine and ethanolamine) on blastocyst expansion in terms of their dose-response relationship.

\section{Materials and Methods}

Embryos. Embryos at the early morula stage were collected from the oviducts of superovulated New Zealand White does $44.48 \mathrm{~h}$ after hCG injection and insemination (Kane, 1987). Embryos were placed in culture in 0.5 ml droplets of medium under paraffin liquid (Art. 7174: Merck, Darmstadt, West Germany) in tissue culture dishes (experiments involving inositol) or in $1 \mathrm{ml}$ medium in 24-well culture dishes and covered with $0.2 \mathrm{ml}$ paraffin liquid (all other experiments). At the end of 5 days in culture (all experiments) the proportion of blastocysts was recorded and embryo diameters were measured with an eyepiece micrometer. In some experiments (those with serine, choline and ethanolamine) the embryo diameters were also measured after 7 days of culture.

Culture medium. The composition of the basic culture medium for all experiments was $0.5 \%$ bovine serum albumin (Cat. no. A4503, lot no. 120F-0089: Sigma, Poole, Dorset, UK), $108 \mathrm{~mm}-\mathrm{NaCl}, 4 \cdot 78 \mathrm{~mm}-\mathrm{KCl}, 1 \cdot 71 \mathrm{~mm}-\mathrm{CaCl} \cdot 2 \mathrm{H}{ }_{2} \mathrm{O}$, $1.19 \mathrm{mM}-\mathrm{KH}_{2} \mathrm{PO}_{4}, 1.19 \mathrm{~mm}-\mathrm{MgSO}_{4} \cdot 7 \mathrm{H}_{2} \mathrm{O}, 25 \mathrm{~mm}-\mathrm{NaHCO}_{3}, 0.5 \mathrm{~mm}$-sodium pyruvate, $1 \mathrm{~mm}$-glucose and the amino acids, vitamins and trace elements of Ham's F10 medium (Ham, 1963; Kane \& Foote, 1970). In experiments in which the effects of inositol, choline and serine (all normal constituents of the basic medium) were being examined, the constituent under study was omitted from the basic medium. Ethanolamine is not a constituent of the basic medium.

Experiments. Four experiments were carried out. In Exp. 1 embryos were cultured in media with myo-inositol at concentrations of $0,0 \cdot 12,0 \cdot 6,3,15,75$ and $375 \mu \mathrm{M}$. In Exp. 2 the effects of choline were examined at concentrations of $0,0 \cdot 04,0 \cdot 2,1,5,25$, and $125 \mu \mathrm{M}$. In Exp. 3 the effects of serine were examined at concentrations of $0,4,20,100,500$ and $2500 \mu \mathrm{M}$. In Exp. 4 the effects of ethanolamine were examined at concentrations of $0,0 \cdot 1,1,10,100$ and $1000 \mu \mathrm{M}$. 
Because of the results of Exp. 1, the concentration of inositol used in Exps 2, 3 and 4 was $75 \mu \mathrm{M}$ ( $\times 25$ the concentration found in F10 medium). Because of the results of Exp. 3, the concentration of serine used in Exp. 4 was $300 \mu \mathrm{M}$ $(\times 3$ the concentration in F10).

Statistical considerations. Embryos collected on one day were pooled and divided among the treatments for that day. The embryos collected on one day constituted one replicate of a randomized complete block experiment. The data on blastocyst expansion were analysed by analysis of variance. Data for blastocyst formation in Exp. 3 were analysed by orthogonally partitioned $\chi^{2}(\mathrm{Li}, 1964)$.

\section{Results}

In all experiments except the serine experiment (Exp. 3), the percentage of blastocysts formed in all treatments was in the range 95-100 and therefore data on proportions of embryos forming blastocysts is not presented for three experiments.

There was a very marked and significant effect $(P<0.01)$ of inositol on blastocyst expansion (Fig. 1). In the treatment without inositol blastocysts were observed to form and start to expand but they rapidly shrank to the size of a morula. The optimal concentration of inositol tested was $75 \mu \mathrm{M}$ and this level of inositol caused a $>4$-fold increase in diameter as compared to the control treatment without inositol.

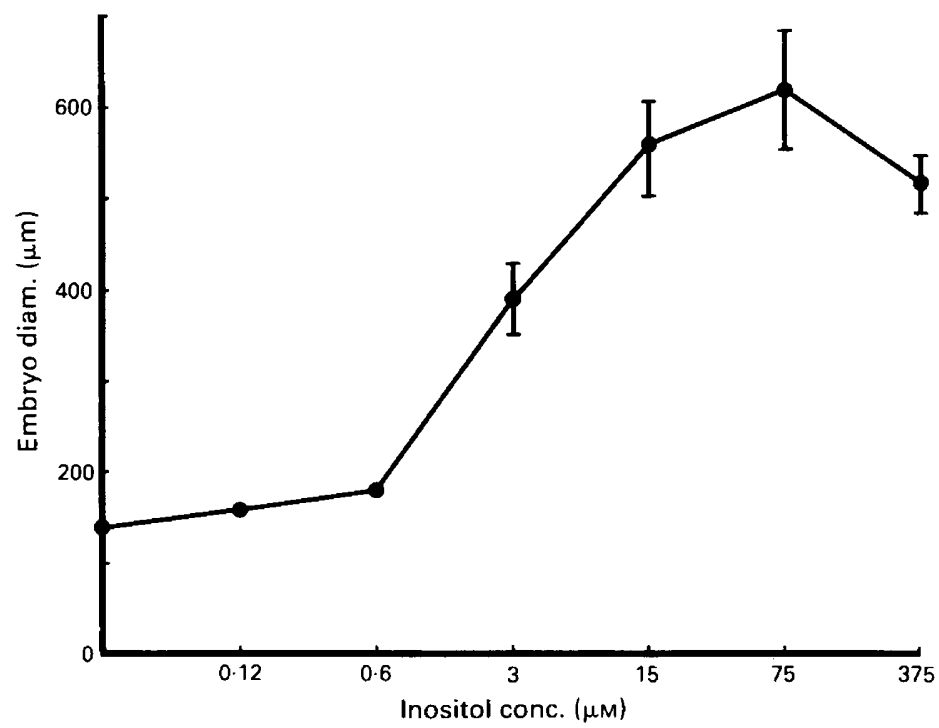

Fig. 1. The effects of myo-inositol on expansion of rabbit blastocysts cultured from the morula stage for 5 days. Values are means \pm s.e.m. with 12-17 embryos/dose level and 3 replicates.

There was also a very marked and significant $(P<0.01)$ effect of serine on blastocyst formation and on blastocyst expansion after both 5 and 7 days of culture (Fig. 2). In the absence of serine, there was a complete absence of blastocyst formation as compared with around $100 \%$ in the treatments with $\geqslant 20 \mu \mathrm{M}$ serine. The optimal concentration of serine tested for blastocyst expansion was $500 \mu \mathrm{M}$ and this concentration resulted in a $>6$-fold increase in diameter after 5 days' culture and a $>10$-fold increase after 7 days' culture as compared with the control treatment without serine.

The results with choline and ethanolamine are presented in Fig. 3. The only significant effect of either compound was that the highest concentration of ethanolamine $(1 \mathrm{~mm})$ significantly depressed blastocyst expansion after 7 days of culture. 


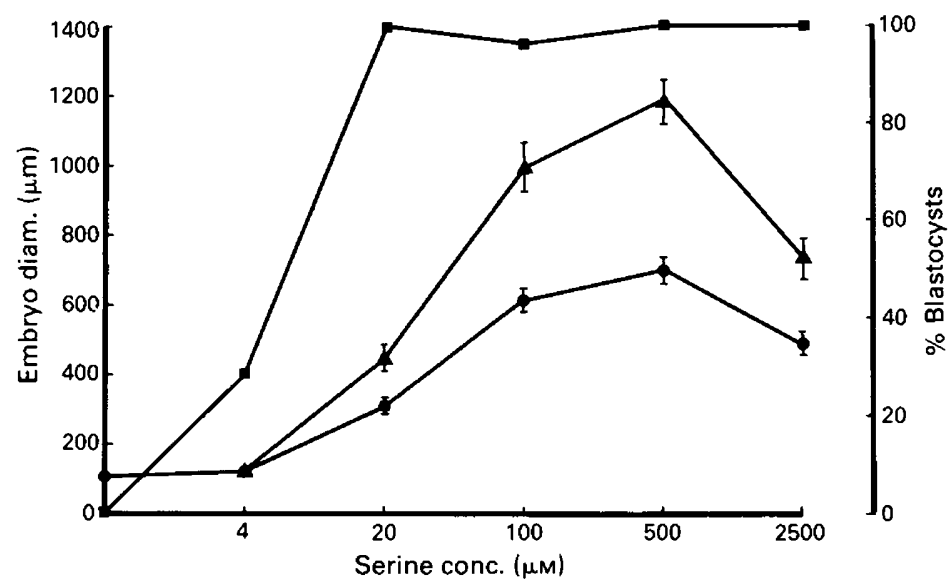

Fig. 2. The effects of serine on development of rabbit morulae to blastocysts ( $\boldsymbol{\square})$ and expansion of blastocysts after 5 days $(\boldsymbol{O})$ and 7 days $(\boldsymbol{\Delta})$ of in-vitro culture. Values for blastocyst expansion are means \pm s.e.m. with 28-29 embryos/dose level, for 2 replicates.

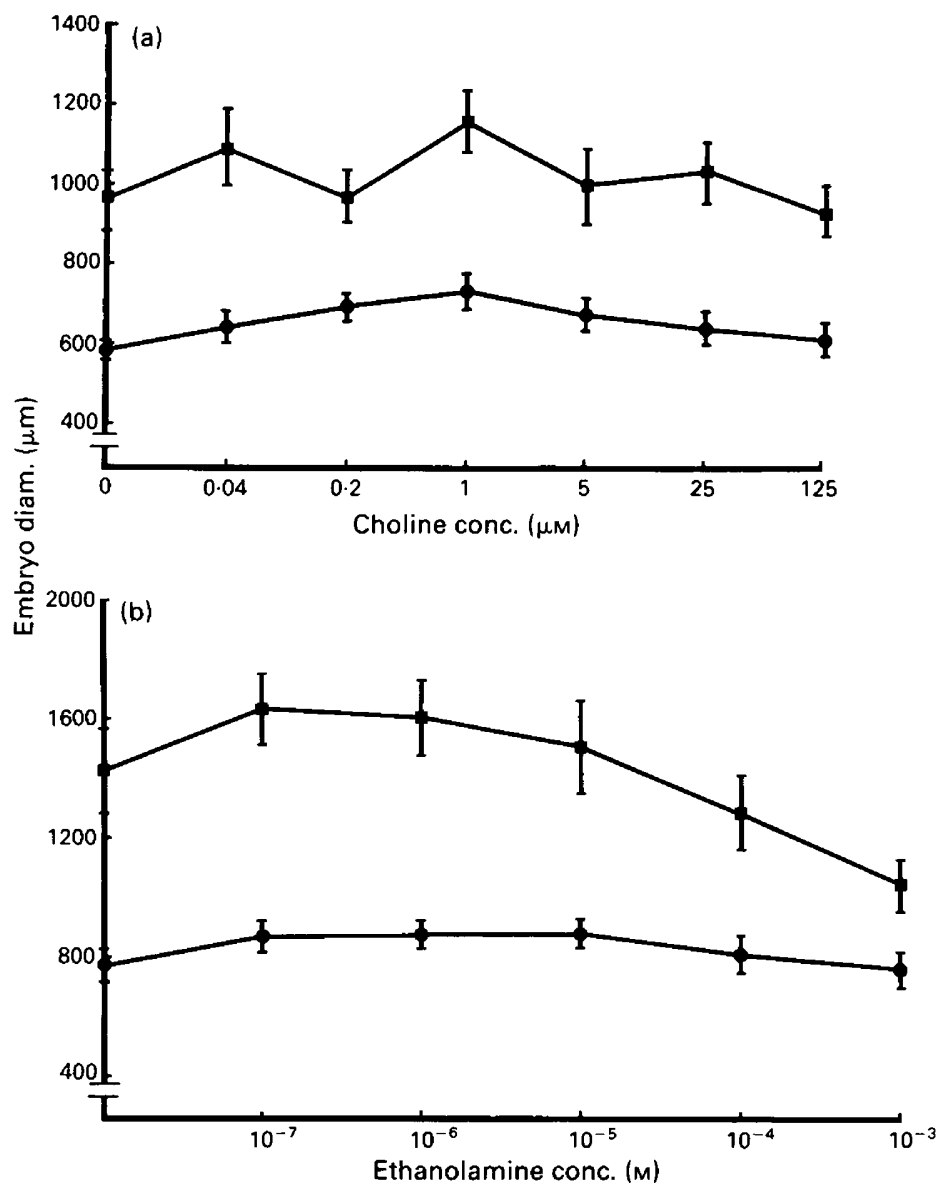

Fig. 3. The effects of (a) choline chloride and (b) ethanolamine on expansion of rabbit blastocysts cultured from the morula stage for 5 days $(\boldsymbol{O})$ and 7 days $(\boldsymbol{\square})$. Values are means \pm s.e.m. for (a) 32-38 embryos/dose level, 7 replicates and (b) 24-27 embryos dose level, 7 replicates. 


\section{Discussion}

The present experiments demonstrate a clear requirement both for inositol and serine for blastocyst growth in the rabbit. There is no evidence for such a requirement for preimplantation growth in the mouse embryo, the pre-eminent current model for studies on preimplantation mammalian development. The most likely explanation for the difference in nutrient requirements between the rabbit and mouse embryo is the difference in preimplantation growth patterns. The mouse embryo does not grow during the preimplantation period: the mouse blastocyst contains only about 20 ng protein which is less than it does at the one-cell stage (Brinster, 1967). In striking contrast, the rabbit blastocyst before implantation contains about $200 \mu \mathrm{g}$ protein (Lutwak-Mann, 1971).

These experiments confirm the finding of Kane (1988) that inositol is essential for culture of rabbit morulae to expanded blastocysts. It is also clear that the concentration $(3 \mu \mathrm{M})$ in Ham's F10 medium (Ham, 1963) is very much below the optimal concentration $(75 \mu \mathrm{M})$ found in these studies. Gregoire et al. (1962) reported concentrations of inositol in rabbit oviducal fluid of $2.6 \mathrm{mg} / 100 \mathrm{ml}$ $(144 \mu \mathrm{M})$ and concentrations in uterine fluid approximately 3 times that figure. Inositol is clearly necessary for rabbit blastocyst growth and the effect on blastocyst diameter is not just the stimulation of blastocyst inflation by some mechanism such as ion pumping. We have shown in preliminary studies that inositol stimulates the incorporation of radiolabelled thymidine and amino acids into rabbit blastocysts (M. Fahy \& M. T. Kane, unpublished observations). We have also found (Kane \& Bavister, 1988) that inositol is essential for hatching of hamster blastocysts cultured from the 8-cell stage in vitro.

There appears to be no information available to date on the role of inositol in preimplantation mammalian development. Inositol is known to be essential in cells for the synthesis of certain membrane phospholipids (Hawthorne, 1982; Vance, 1986) and it is probable that at the blastocyst stage it becomes necessary for the synthesis of phospholipid components of new membranes. There is an accumulation of evidence over the past 20 years showing a correlation between metabolism of phosphatidylinositol and other phosphoinositides, and cell stimulation and proliferation (reviewed by Nishizuka, 1984; Berridge, 1987a, b). As yet no one has demonstrated a role for this phosphatidylinositol second messenger system in preimplantation mammalian embryos, but in preliminary experiments we have been able to show that radiolabelled inositol is incorporated into phosphatidylinositol by rabbit blastocysts (M. Fahy, E. R. S. Roldan, R. A. P. Harrison \& M. T. Kane, unpublished data). Irvine \& Moor (1986) have shown that this system is involved in activation of sea-urchin eggs.

These experiments demonstrate an acute requirement in rabbit embryos for serine for both blastocyst formation and expansion. This requirement is shown most clearly by the fact that, even in the presence of a full supply of the other 19 amino acids, the omission of serine prevented all morulae without exception from developing to blastocysts. Gregoire et al. (1961) reported levels of serine of $1.1 \mathrm{ng} / 100 \mathrm{ml}(105 \mu \mathrm{M})$ in rabbit oviducal fluid and of $10.4 \mathrm{mg} / 100 \mathrm{ml}(990 \mu \mathrm{M})$ in uterine fluid and the optimal tested concentration in these experiments $(500 \mu \mathrm{M})$ falls between these two figures.

Serine is known to play a number of essential roles in cells as well as the obvious one of being a precursor for protein synthesis. It is necessary for synthesis of the phospholipid, phosphatidylserine (Ansell \& Spanner, 1982). This phospholipid is involved in function of the phosphatidylinositol second messenger system (Berridge, 1987a). Serine is also involved in synthesis of purines for nucleic acid synthesis by a de-novo pathway (Hartman, 1970). The drastic effect of omission of serine on rabbit blastocyst formation and expansion may be due to interference with one or more of these roles.

The fact that there was no significant effect of choline is perhaps surprising in view of its importance as a component of phospholipids (Ansell \& Spanner, 1982; Vance, 1986). Pratt (1980) has shown in the mouse embryo that choline is incorporated into phosphatidylcholine and lysolecithin. It has also been found that omission of choline from the culture medium inhibits hatching of 
hamster blastocysts (Kane \& Bavister, 1988). Choline can be synthesized from the amino acid serine (Ansell \& Spanner, 1982) and it is possible that rabbit embryos have the ability to do this from the serine present in the culture medium.

The only significant effect of ethanolamine was to depress blastocyst expansion at the highest concentration tested. While ethanolamine as phosphatidylethanolamine is an important component of cellular phospholipids (Ansell \& Spanner, 1982), it is normally synthesized from serine and it is probable that the rabbit embryo is able to do this from the serine in the culture medium.

Definitive proof that the requirement for inositol and serine for rabbit blastocyst growth shown in these experiments is related to phospholipid synthesis must await further studies on the incorporation of radiolabelled inositol and serine.

I thank the Irish Health Research Board and the SmithKline Foundation for research support.

\section{References}

Ansell, G.B. \& Spanner, S. (1982) Phosphatidylserine phosphatidylethanolamine and phosphatidylcholine. In Phospholipids, pp. 1-49. Eds J. N. Hawthorne \& G. B. Ansell. Elsevier Biomedical Press, Amsterdam.

Berridge, M.J. (1987a) Inositol triphosphate and diacylglycerol: two interacting second messengers. Ann. Rev. Biochem. 56, 159-193.

Berridge, M.J. (1987b) Inositol lipids and cell proliferation. Biochim. Biophys. Acta 907, 33-45.

Brinster, R.L. (1967) Protein content of the mouse embryo during the first five days of development. $J$. Reprod. Fert. 13, 413-420.

Daniel, J.C., Jr (1967) Vitamins and growth factors in the nutrition of rabbit blastocysts in vitro. Growth 31, $71-77$.

Gregoire, A.T., Gongsakdi, D. \& Rakoff, A.E. (1961) the free amino acid content of the female rabbit genital tract. Fert. Steril. 12, 322-327

Gregoire, A.T., Gongsakdi, D. \& Rakoff, A.E. (1962) The presence of inositol in genital tract secretions of the female rabbit. Fert. Steril. 13, 432-435.

Ham, R.G. (1963) An improved nutrient solution for diploid Chinese hamster and human cell lines. Expl Cell Res. 29, 515-526.

Hartman, S.C. (1970) Purines and pyrimidines. In Metabolic Pathways: Vol. IV, Nucleic Acids, Protein Synthesis, and Coenzymes, pp. 1-68. Ed. D. M. Greenberg. Academic Press, New York.

Hawthorne, J.N. (1982) Inositol phospholipids. In Phospholipids, pp. 263-278. Eds J. N. Hawthorne \& G. B. Ansell. Elsevier Biomedical Press, Amsterdam.

Irvine, R.F. \& Moor, R.M. (1986) Micro-injection of inositol 1,3,4,5-tetrakisphosphate activates sea urchin eggs by a mechanism dependent on external $\mathrm{Ca}^{2+}$. Biochem. J. 240, 917-920.
Kane, M.T. (1987) In vitro growth of preimplantation rabbit embryos. In The Mammalian Preimplantation Embryo: Regulation of Growth and Differentiation in Vitro, pp. 193-217. Ed. B. D. Bavister. Plenum Press, New York.

Kane, M.T. (1988) The effects of water-soluble vitamins on the expansion of rabbit blastocysts in vitro. $J$. expl. Zool. 245, 220-223.

Kane, M.T. \& Bavister, B.D. (1988) Vitamin requirements for development of eight-cell hamster embryos to hatching blastocysts in vitro. Biol. Reprod. 39, 1137-1143.

Kane, M.T. \& Foote, R.H. (1970) Culture of two- and four-cell rabbit embryos to the expanding blastocyst stage in synthetic media. Proc. Soc. exp. Biol. Med. 133, $921-925$.

Li, J.C.R. (1964) Statistical Inference, Vol. 1. Edward Bros., Ann Arbor.

Lutwak-Mann, C. (1971) The rabbit blastocyst and its environment: Physiological and biochemical aspects. In The Biology of the Blastocyst, pp. 243-260. Ed. R. J. Blandau. University of Chicago Press, Chicago.

Nishizuka, Y. (1984) The role of protein kinase C in cell surface signal transduction and tumour promotion. Nature, Lond. 308, 693-698.

Pratt H.P.M. (1980) Phospholipid synthesis in the preimplantation mouse embryo. J. Reprod. Fert. 58, 237-248.

Vance, D.E. (1986) Phospholipid metabolism in eucaryotes. In Biochemistry of Lipids and Membranes, pp. 242-270. Eds D. E. Vance \& J. Vance. The Benjamin/ Cummings Publishing Co., Inc., Menlo Park.

Received 25 January 1989 